\begin{tabular}{|c|c|c|c|c|}
\hline $\mathrm{Nr}$ & & geschätzte & Grösse & I 2.0 \\
\hline$\gg$ & 16 & » & 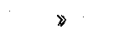 & I 3.3 \\
\hline \# & 18 & 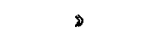 & $\nabla$ & 13.0 \\
\hline , & 19 & 》 & 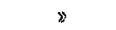 & 13.0 \\
\hline$\gg$ & 20 & \# & $\gg$ & 117 \\
\hline . & 31 & $\gg$ & $D$ & I I . 5 \\
\hline » & $3^{6}$ & " & $D$ & I 30 \\
\hline & 39 & 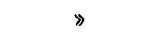 & $D$ & 11.5 \\
\hline
\end{tabular}

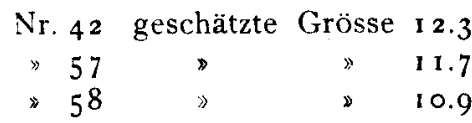

Der Stern 58 ist durch den in der Nähe stehender Stern 60, der wie alle helleren Sterne auf der Photographie sehr lang gezogen ist, verdeckt worden. Zweifelhaft ist das Vorhandensein des Sterns 32, Gr. I1.3, auf der Photographie.

\title{
Notiz über die Eigenbewegung von Lal. 18069.
}

Der Stern Lal. I 8069, auf dessen Eigenbewegung Dr. Kreutz in Nr. 2768 der A. N. S. I 25 auf eine Anfrage von Herrn J. Tebbutt aufmerksam machte, wurde in Wien bei der Wiederbeobachtung der südlichen Santini'schen Zonen dreimal beobachtet. Die auf 1885.0 reducirten Positionen desselben lauten:

$\begin{array}{rrr}\text { I } 882 & \text { Febr. I I } \alpha=9^{\mathrm{h}} 3^{\mathrm{m}} 19^{5.06} \delta=-10^{\circ} \text { I } 7^{\prime} 19.8 \\ 1885 \text { April I } & 18.63 & 20.7 \\ 1887 \text { Jan. 27 } & 18.90 & 20.3\end{array}$

Die mittlere Beobachtung ist unter sehr ungünstigen Verhältnissen angestellt, daher wohl am besten ganz unberücksichtigt zu lassen.

Reducirt man die Lalande'sche Position mit v. Asten's, die Bessel'sche mit den neuen Königsberger Hülfstafeln, und bringt man alles mit der Struve'schen Präcessionsconstante auf 1875.0 , so erhält man, ohne Anfügen irgend einer systematischen Correction, folgende Oerter:

\begin{tabular}{|c|c|c|c|c|c|c|}
\hline & & $\alpha$ & & $\delta$ & $\mathrm{Bb}$. & Ep. \\
\hline Lal. I 8069 & $7^{\mathrm{m}}$ & $9^{h} 2^{m} 51^{5} \cdot 92$ & $-10^{\circ}$ & I $4^{\prime} 4^{\prime \prime \prime} 8$ & I & 1798.2 \\
\hline B.Z. Z. 217 & 7.8 & $5^{1} .53$ & & $55 \cdot I$ & I & 1824.1 \\
\hline Sant $_{2} V .205$ & 8 & 50.75 & & 53.6 & 2 & I 845.3 \\
\hline Wien Mer. & - & 49.95 & & $5^{6.4}$ & 2 & 1884.6 \\
\hline
\end{tabular}

Eine AR.: $9^{\mathrm{h}} 2^{\mathrm{m}} 50^{\mathrm{s}} \mathrm{I} 9$ für 1875.0 mit einer jährlichen E. B.: $\Delta \alpha=-0.024$ bringt diese Oerter in eine befriedigende Uebereinstimmung. In Declination ist die E. B. jedenfalls sehr gering.

Währing 1887 Febr. 4

\section{E. Weiss.}

\section{Sonnen-Statistik für $\mathbf{1} 886$.}

Nach den Zählungen der Gruppen und Flecken der Sonne auf der Zürcher Sternwarte, und den magnetischen Variations-Beobachtungen in Mailand, ergeben sich für die Sonnenflecken-Relativzahlen $r$, die Declinations-Variationen $v$, und die Vermehrungen $\Delta r$ und $\Delta v$ dieser beiden Zahlen gegenüber den correspondirenden Epochen von 1885 folgende Werthe:

\begin{tabular}{l|r|r|r|r}
\hline I886 & $r$ & $v$ & $\Delta r$ & $\Delta v$ \\
\hline Jan. & 28.4 & 4.07 & -3.0 & +0.18 \\
Febr. & 23.6 & $4.9 \mathrm{I}$ & -43.6 & +0.16 \\
März & 61.8 & 8.61 & +15.2 & -0.22 \\
April & 45.9 & 9.89 & -8.7 & -0.75 \\
Mai & 29.0 & 9.06 & -51.5 & $-\mathbf{1} .40$ \\
Juni & 25.7 & 8.37 & -56.4 & -3.67 \\
Juli & 32.9 & 9.58 & -28.5 & -1.20 \\
Aug. & $\mathbf{1} 9.0$ & 8.17 & -28.7 & -1.96 \\
Sept. & 17.1 & 7.61 & -26.3 & -1.71 \\
Oct. & 9.5 & 6.33 & -33.1 & -0.91 \\
Nov. & 0.0 & 2.48 & -26.8 & $-\mathbf{1 . 9 2}$ \\
Dec. & $\mathbf{1 5 . 1}$ & 1.61 & -3.8 & $--\mathbf{1 . 2 6}$ \\
\hline Jahr & $\mathbf{2 5 . 7}$ & 6.72 & -24.6 & $-\mathbf{1 . 2 3}$
\end{tabular}

Es haben sich also sowohl Relativzahl als Variation auch in diesem Jahre wieder stark vermindert, und zwar in ganz entsprechender Weise; denn wenn man die mittlere Relativzahl $r=25.7$ in die für Mailand vor Jahren von mir abgeleitete Formel

$$
v=5: 62+0.045 \cdot r
$$

einführt, so erhält man $v=6: 79$, also eine Zahl, welche nur um 7 Hundertstel von der aus den magnetischen Beobachtungen direct abgeleiteten Zahl $v=6: 72$ abweicht. Es hat sich also der merkwürdige Rapport zwischen den beiden Erscheinungen wieder glänzend bestätigt.

Zürich 1887 Jan. 12.

R. Wolf. 